\title{
Estimation de l'exhaustivité des données de facturation des médecins pour la détermination des cas de diabète au moyen des données sur les médicaments d'ordonnance
}

\author{
L. M. Lix, Ph. D. (1); J. P. Kuwornu, M. Sc. (1); K. Kroeker, B. Sc. (1); G. Kephart, Ph. D. (2); K. C. Sikdar, Ph. D. (3);
} M. Smith, M. Sc. (4); H. Quan, Ph. D. $(3,5)$

Cet article a fait l'objet d'une évaluation par les pairs.

Diffuser cet article sur Twitter

\section{Résumé}

Introduction : Les changements dans les politiques de remboursement des médecins peuvent faire obstacle à la collecte des données administratives de facturation des médecins, ce qui pourrait mener à des estimations biaisées de la prévalence et de l'incidence des maladies. L'ampleur de cette perte potentielle de données demeure cependant largement inconnue. Notre étude vise à estimer l'exhaustivité de la couverture des cas de maladie pour le Manitoba par les médecins rémunérés à l'acte (RA) et par les médecins non rémunérés à l'acte (NRA).

Méthodes : Les données administratives du Manitoba ont servi à créer une cohorte dont les membres (20 ans et plus) devaient avoir pris un nouveau médicament pour le diabète entre le $1^{\text {er }}$ avril 2007 et le 31 mars 2009. Ces membres ont été classés par mode de paiement du médecin prescripteur (c'est-à-dire rémunérés à l'acte ou non rémunérés à l'acte), puis selon s'ils avaient ou non reçu de diagnostic de diabète d'après les données de facturation des médecins et des dossiers d'hôpitaux. On a utilisé des analyses statistiques $\chi^{2}$ pour tester les différences de caractéristiques entre les deux groupes.

Résultats : La cohorte se composait de 12394 individus, dont 86,4 \% avaient reçu une ordonnance de médicament pour le diabète d'un médecin RA. Au total, 1172 médecins ( $81,8 \%$ RA) ont prescrit ce type de médicaments pour la cohorte. Les membres de la cohorte ayant reçu une ordonnance d'un médecin RA étaient plus âgés et plus susceptibles de résider dans la région sanitaire de Winnipeg que ceux qui avaient reçu une ordonnance d'un médecin NRA. Un plus grand pourcentage de cas traités par des médecins NRA n'avait pas reçu de diagnostic de diabète (18,7 \%, contre 14,9 \% pour les médecins RA).

Conclusion : Les résultats suggèrent une perte minimale de données de facturation des médecins associée aux politiques de rémunération au Manitoba. Cette méthode d'évaluation de l'exhaustivité des données pourrait être appliquée à d'autres maladies chroniques et à d'autres secteurs de compétence.

Mots-clés : maladies chroniques, dossiers médicaux, surveillance, qualité des données

\section{Introduction}

Les données administratives électroniques sur la santé sont largement utilisées au Canada et ailleurs dans le monde pour mener des recherches et exercer une surveillance sur les maladies chroniques à l'échelle des populations ${ }^{1,2}$. Ces données sont appréciées pour plusieurs raisons : elles sont à jour, elles contiennent des
Points saillants

- Les données de facturation des médecins sont utiles pour la recherche et la surveillance relatives à la santé des Canadiens.

- Il y a davantage de médecins payés de diverses façons, et nombreux sont ceux qui passent d'une rémunération à l'acte (RA) à d'autres formes de rémunération (NRA). Les données de facturation des médecins peuvent ne pas comporter d'information sur les contacts avec leurs patients dans le cas des médecins NRA.

- Nous avons utilisé les dossiers sur les médicaments d'ordonnance prescrits aux patients diabétiques pour estimer l'exhaustivité des données de facturation des médecins au Manitoba.

- $14,9 \%$ des personnes ayant reçu une ordonnance pour le diabète d'un médecin RA n'avaient pas obtenu de diagnostic de diabète au cours des deux années précédant et des deux années suivant cette ordonnance.

- $18,7 \%$ des personnes ayant reçu une ordonnance pour le diabète d'un médecin NRA n'avaient pas obtenu de diagnostic de diabète au cours des deux années précédant et des deux années suivant l'ordonnance.

- Les résultats suggèrent que la perte de données en raison de données de facturation manquantes est faible. Cependant, cette perte signifie que l'incidence est sous-estimée.

Rattachement des auteurs :

1. Département des sciences de la santé communautaire, Université du Manitoba, Winnipeg (Manitoba), Canada

2. Département de santé communautaire et d'épidémiologie, Université Dalhousie, Halifax (Nouvelle-Écosse), Canada

3. Institut de cardiologie Libin de l'Alberta, Université de Calgary, Calgary (Alberta), Canada

4. Centre d'élaboration de la politique des soins de santé du Manitoba, Université du Manitoba, Winnipeg (Manitoba), Canada

5. Département de sciences de la santé communautaire, Université de Calgary, Calgary (Alberta), Canada

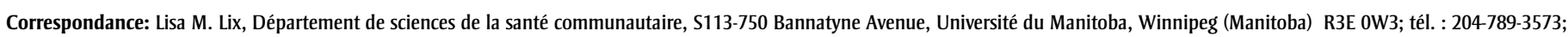
téléc. : 204-789-3905; courriel : lisa.lix@med.umanitoba.ca 
renseignements sur de nombreuses personnes et elles sont relativement peu coûteuses d'accès et d'utilisation. Les données électroniques de facturation des médecins, qui correspondent aux dossiers de facturation ou aux factures relatives aux consultations externes, constituent une importante source d'information pour la recherche et la surveillance, parce que la grande majorité des personnes ayant une maladie chronique consulte régulièrement son médecin pour la gestion et le traitement de sa maladie.

Toutefois, la qualité des données administratives sur la santé disponibles pour la recherche et la surveillance a été remise en question, ce qui a entraîné un certain nombre d'études sur le sujet. La plupart de ces études ont porté sur la validité de l'information diagnostique ${ }^{3-6}$, alors que plusieurs autres aspects de la qualité doivent également être évalués pour s'assurer que les données sont adaptées à la recherche et à la surveillance. Par exemple, il est essentiel de recueillir des données complètes sur une population pour garantir que les recherches et les résultats de surveillance sont objectifs. Des lacunes en matière d'intégralité des données peuvent entraîner des problèmes d'estimation semblables à ceux rencontrés avec les biais de non-réponse dans les données d'enquête ${ }^{7}$.

Les renseignements contenus dans les données de facturation des médecins sont utilisés pour rémunérer les médecins rémunérés à l'acte (RA) pour les services qu'ils fournissent aux patients. Les médecins non rémunérés à l'acte (NRA), qui reçoivent un salaire ou sont payés par contrat, utilisent souvent un processus de facturation fictive dans lequel ils soumettent des factures parallèles. Toutefois, ce processus ne permet pas toujours de saisir systématiquement les renseignements sur les services de santé fournis. Une étude de 2009 a rapporté une sous-estimation de l'incidence du diabète en raison de la nature incomplète des données de facturation fictives de l'Ontario ${ }^{8}$. Cependant, l'ampleur de ce problème reste inconnue. Les systèmes de soins de santé nationaux et internationaux adoptant de plus en plus de nouveaux modèles de prestation des services de soins primaires et de nouveaux modes de paiement des médecins, la cueillette de données complètes sur les services rendus par les médecins est menacée?

Le but de notre étude était d'estimer le degré d'exhaustivité de la cueillette de données sur les cas de maladie chronique associée à la facturation fictive manquante des médecins NRA dans les données administratives sur la santé. Notre étude porte spécifiquement sur le diabète car les codes de diagnostic dans les données administratives ont offert une bonne sensibilité et une bonne spécificité pour la détermination des cas de cette maladie et que la surveillance du diabète à l'aide de données administratives électroniques est une question qui suscite de l'intérêt dans le monde entire ${ }^{5}$.

\section{Méthode}

\section{Sources de données}

Nous avons utilisé des données de la province du Manitoba, dont la population est d'environ 1,2 million de personnes selon le recensement de Statistique Canada de 2011. Le Manitoba Centre for Health Policy abrite de nombreuses bases de données administratives électroniques pouvant être jumelées en préservant l'anonymat au moyen d'un numéro d'identification personnelle de santé (NIPS) unique. Le Manitoba, comme d'autres provinces canadiennes, offre un système universel de soins de santé, ce qui signifie que pratiquement toute la population de cette province est inscrite pour recevoir des services de soins de santé.

Les médecins RA soumettent toutes leurs factures au ministère de la Santé de la province. Les médecins rémunérés selon d'autres méthodes (NRA) sont tenus de soumettre des factures fictives en parallèle au ministère de la Santé, mais on ignore dans quelle mesure cette exigence est respectée, puisque la rémunération de ces médecins ne dépend pas de cette présentation de factures et que les réclamations fictives ne font habituellement pas l'objet d'un audit. Un diagnostic unique est enregistré pour chaque réclamation à l'aide des codes de la Classification Internationale des Maladies, $9^{e}$ révision, modification clinique (CIM-9-CM). Les registres de congés des hôpitaux, qui sont remplis lorsqu'un patient sort d'un établissement de soins actifs, contiennent des diagnostics enregistrés à l'aide des codes de la Classification Internationale des Maladies, $10^{e}$ révision, version canadienne (CIM-10-CA). Chaque enregistrement contient jusqu'à 25 diagnostics. Les dossiers sur les médicaments d'ordonnance proviennent du Réseau pharmaceutique informatisé (RPI), une base de données électronique centralisée de points de vente reliant toutes les pharmacies de détail du Manitoba. Pour chaque prescription remplie, de l'information est recueillie, notamment la date, les caractéristiques du médicament (comme la posologie et le numéro d'identification du médicament) et le numéro d'identification du prescripteur. Le registre de population contient les dates de couverture de l'assurance maladie et des données personnelles telles que la date de naissance, le sexe et le lieu de résidence. Le registre des fournisseurs contient des instantanés trimestriels des caractéristiques des médecins ainsi que leur date de naissance, leur spécialité, leur lieu de pratique, leur numéro d'identification de fournisseur et leurs modalités de paiement. Nous avons utilisé la mise à jour la plus récente du registre pour notre étude, celle concernant le premier trimestre (janvier à mars) 2009.

Les données administratives sur la santé du Manitoba ont déjà été largement utilisées dans le cadre de recherches sur les maladies chroniques et d'études de surveillance ${ }^{10-12}$.

Nous avons reçu l'approbation éthique du Comité d'éthique de la recherche en santé de l'Université du Manitoba ainsi que la permission d'accéder aux données de l'étude par le Comité de la protection des renseignements médicaux du Manitoba.

\section{Cohorte étudiée}

Nous avons monté une cohorte de cas incidents de diabète diagnostiqués chez les adultes à partir des dossiers sur les médicaments d'ordonnance, dossiers qui ont démontré une excellente sensibilité pour la détermination des cas de diabète ${ }^{13}$. Les critères d'inclusion dans cette cohorte étaient : 1) au moins une ordonnance avec 
le code A10 de la classification Anatomique Thérapeutique Chimique (ATC) (soit les médicaments pour le diabète) durant la période d'observation de deux ans couvrant les exercices 2007-2008 et 2008-2009 (un exercice s'étendant du $1^{\text {er }}$ avril au 31 mars), 2) une couverture d'assurance continue pendant la période de deux ans avant et la période de deux ans après la date de l'ordonnance de référence, c'està-dire la date à laquelle un médicament prescrit pour le diabète a été entré pour la première fois dans la base de données du RPI au cours de la période d'observation et 3) être âgé d'au moins 20 ans révolus à la date de l'ordonnance de référence. Les individus étaient exclus de l'étude s'ils avaient reçu une ordonnance portant le code A10 de la classification ATC dans les 730 jours précédant la date de cette ordonnance de référence.

Le code A10 de la classification ATC concerne les hypoglycémiants, tels que la metformine, les insulines et les médicaments similaires, mais ne s'applique pas aux fournitures comme les bandelettes de test glycémique. Une étude de 2009 a utilisé une période d'un an pour exclure les cas non incidents ${ }^{8}$, mais nous avons étendu cette période à deux ans afin d'accroître la probabilité que les membres de la cohorte aient reçu une ordonnance concernant un médicament pour le diabète pour la première fois.

Le numéro d'identification du prescripteur associé à l'ordonnance de référence de médicaments pour le diabète a été jumelé, en respectant l'anonymat, au numéro correspondant dans le registre des fournisseurs de soins, afin de déterminer le mode de paiement du médecin (RA ou RNA). On a exclu les individus lorsque le mode de paiement de leur fournisseur de soins ayant fait leur ordonnance de référence n'avait pas été consigné dans le registre. Les membres de la cohorte ont été répartis en deux groupes mutuellement exclusifs : ceux dont l'ordonnance de référence provenait d'un médecin RA et ceux dont l'ordonnance de référence provenait d'un médecin RNA.

\section{Variables à l'étude}

L'identification des diagnostics de diabète dans les données administratives a été faite à l'aide de la définition de cas de diabète du Système national de surveillance des maladies chroniques (SNSMC), selon laquelle une hospitalisation ou deux réclamations de facturation d'un médecin portant le code CIM-9 250.00 ou le code E10-E14 de la CIM-10-CA sont requises en deux $a^{4} s^{4,14}$. La date de diagnostic du diabète est la date d'enregistrement de ce diagnostic dans les dossiers d'hôpital ou la date de premier diagnostic dans les dossiers de facturation d'un médecin. Les membres de la cohorte répondant aux exigences de la définition de cas et pour qui le diagnostic de diabète a été posé entre deux ans avant et deux ans après la date de l'ordonnance de référence ont été classés comme des cas inclus. Tous les autres ont été classés comme des cas non inclus.

La cohorte a été caractérisée par son profil sociodémographique : groupe d'âge, sexe, quintile de revenu et région sanitaire de résidence. Deux groupes d'âge ont été distingués, les moins de 65 ans et les 65 ans et plus. Le quintile de revenu est une mesure spatialisée basée sur le revenu moyen des ménages selon le recensement de Statistique Canada. Le code postal des individus extrait du registre de population est associé à une aire de diffusion (AD), la plus petite unité géographique de diffusion des données du recensement. La population du Manitoba a ensuite été répartie en cinq groupes à peu près égaux selon le revenu moyen des ménages de leur $A D^{15}$, séparément pour les populations urbaines et rurales. La région sanitaire de résidence a été définie comme étant Winnipeg ou autre que Winnipeg. Cette dernière englobe quatre régions sanitaires rurales : Entreles-Lacs et de l'Est, Nord, Prairies Mountain et Santé Sud. Les réserves des Premières Nations sont principalement situées à l'extérieur de la région sanitaire de Winnipeg. Toutes les mesures des caractéristiques sociodémographiques ont été prises à la date de l'ordonnance de référence.

Étant donné que les patients avec un diabète plus avancé (c'est-à-dire plus de comorbidité) étaient plus susceptibles d'être détectés au moyen de la définition de cas du Système national de surveillance des maladies chroniques $(\mathrm{SNSMC})^{8}$, nous avons examiné la prévalence de certaines conditions de comorbidité cardiovasculaire dans la cohorte à l'aide des diagnostics figurant dans les registres de congés des hôpitaux. Ces conditions comprenaient l'hypertension (CIM-10-CA I10-I13, I15), l'insuffisance cardiaque congestive (CIM10-CA J50), les syndromes coronariens aigus (CIM-10-CA J21, J22, J23, J24.9, J20.0, J20.1), les maladies cérébrovasculaires, l'accident vasculaire cérébral (p. ex. : CIM-10-CA J60, J61, J63, J64) et la fibrillation auriculaire (CIM-10-CA I48). Toutefois, puisque la fréquence était assez faible pour certains états de santé, nous les avons classés dans la catégorie « hypertension uniquement » et " toutes les conditions de comorbidité cardiovasculaire ».

Les médecins qui ont prescrit des médicaments pour le diabète aux membres de la cohorte de l'étude ont été caractérisés par leur sexe, leur groupe d'âge (moins de 35 ans, 35-60 ans ou 61 ans et plus), leur région sanitaire de pratique (Winnipeg ou autre que Winnipeg) et leur spécialité (spécialiste ou généraliste). La mesure de toutes les caractéristiques des médecins a également été effectuée à la date de l'ordonnance de référence.

\section{Analyses statistiques}

Nous avons décrit la cohorte d'étude et leurs médecins prescripteurs en utilisant des fréquences et des pourcentages. Un test du $\chi^{2}$ a été utilisé pour déterminer les différences entre les caractéristiques du groupe de médecins RA et celles du groupe de médecins NRA. Nous avons estimé le pourcentage de membres de la cohorte dont le diagnostic de diabète était manquant et pour la cohorte dans son ensemble, et par groupe d'âge.

Nous avons estimé le taux d'incidence brut du diabète pour la population adulte (20 ans et plus) du Manitoba en 2007-2008 et 2008-2009 à l'aide de la définition de cas du SNSMC. Le dénominateur était la population totale d'adultes du registre de l'état civil du Manitoba. Nous avons calculé le taux observé et les taux ajustés pour les cas non inclus.

Toutes les analyses ont été effectuées à l'aide du logiciel SAS, version 9.3 (SAS Institute, Cary, Caroline du Nord, États-Unis). 


\section{Résultats}

Au total, 73719 personnes ont reçu une ordonnance pour le diabète en 2007-2008 ou 2008-2009. Après l'application de nos critères d'exclusion (c.-à-d. aucune couverture d'assurance maladie provinciale continue [8,0 \%]; consommation de médicaments pour le diabète durant les 730 jours précédant la date de l'ordonnance de référence [74,3 \%]; moins de 20 ans à la date de l'ordonnance de référence $[0,4 \%]$; ordonnance de référence rédigée par un médecin dont le mode de paiement n'a pu être élucidé [0,5\%]), 12394 personnes ont été maintenues dans la cohorte.

La majorité $(86,4 \%)$ des membres de la cohorte de l'étude ont reçu leur ordonnance de référence d'un médecin RA (tableau 1). En général, les personnes dont l'ordonnance de référence émanait d'un médecin RA étaient plus susceptibles que celles dont l'ordonnance de référence émanait d'un médecin NRA d'être plus âgées, de résider à Winnipeg et de faire partie du quintile de revenu urbain le plus faible ( $p<0,001$ dans tous les cas).

Au total, 1172 médecins ont prescrit des médicaments pour le diabète à au moins un membre de la cohorte durant la période d'observation (tableau 2). En moyenne, chaque médecin RA a remis une ordonnance à 11,2 membres de la cohorte et chaque médecin NRA a remis une ordonnance à 7,9 membres de la cohorte. Par rapport aux médecins NRA, les médecins RA étaient plus susceptibles d'être plus âgés, d'être des spécialistes et de pratiquer à Winnipeg ( $p<0,001$ dans tous les cas). Il n'y avait pas de différence entre le sexe des médecins RA et celui des médecins NRA $(p=0,086)$.

Un pourcentage plus élevé de membres de la cohorte n'ayant pas reçu de diagnostic ont obtenu leur ordonnance de référence d'un médecin NRA plutôt que d'un médecin RA (18,7 \% contre $14,9 \% ; p<0,001$; tableau 3). Dans l'analyse stratifiée selon l'âge, un pourcentage plus élevé de membres de la cohorte âgés de moins de 65 ans n'ayant pas reçu de diagnostic a été observé pour la cohorte NRA par rapport à la cohorte RA $(20,4 \%$ contre $16,5 \%$; $p<0,001)$. Les pourcentages pour les 65 ans et plus n'étaient pas significativement différents ( $p=0,174)$.

Les membres de la cohorte n'ayant pas reçu de diagnostic et dont l'ordonnance de référence provenait d'un médecin NRA étaient plus susceptibles d'être de sexe masculin, de ne pas résider à Winnipeg et d'appartenir au quintile de revenu rural le plus faible, par rapport à ceux dont l'ordonnance de référence provenait d'un médecin RA ( $p<0,001$; tableau 4).

Parmi les membres de la cohorte dont l'ordonnance de référence provenait d'un médecin NR, ceux dont le diagnostic avait été enregistré dans les données administratives étaient plus susceptibles d'avoir été hospitalisés pour des maladies cardiovasculaires comorbides que ceux qui n'avaient pas reçu de diagnostic $(\mathrm{p}<0,001$; tableau 5). De même, les membres de la cohorte NRA dont le diagnostic avait été enregistré étaient plus susceptibles d'avoir été hospitalisés pour maladies cardiovasculaires comorbides que ceux qui n'avaient pas reçu de diagnostic $(p<0,001)$.

En se fondant sur la définition de cas de diabète du SNSMC, 12877 nouveaux cas de diabète adultes (20 ans et plus) diagnostiqués ont été décelés au Manitoba au cours des exercices 2007-2008 et 2008-2009, soit un taux d'incidence brut observé de $1,6 \%$ (figure 1 ). Après ajustement pour tenir compte des cas non inclus des médecins NRA, ce taux est passé à $1,7 \%$, et après ajustement pour tenir compte des cas non inclus des médecins RA et NRA, il est passé à $1,9 \%$.

\section{Analyse}

Dans l'ensemble, les médecins NRA ont prescrit moins de médicaments pour le

TABLEAU 1

Caractéristiques de la cohorte de l'étude, par mode de rémunération du médecin prescripteur

\begin{tabular}{|c|c|c|c|}
\hline \multirow[t]{2}{*}{ Caractéristiques } & \multicolumn{3}{|c|}{ Cohorte } \\
\hline & $\begin{array}{c}\text { Médecins RA } \\
\text { n (\%) }\end{array}$ & $\begin{array}{c}\text { Médecins NRA } \\
\text { n (\%) }\end{array}$ & $\begin{array}{c}\text { Tous les médecins } \\
\text { n (\%) }\end{array}$ \\
\hline Total $^{\mathrm{a}}$ & $10714(86,4)$ & $1680(13,6)$ & $12394(100,0)$ \\
\hline \multicolumn{4}{|l|}{ Groupe d'âge (ans) $^{* *}$} \\
\hline $20-64$ & $7733(72,2)$ & $1305(77,7)$ & $9038(72,9)$ \\
\hline 65 et plus & $2981(27,8)$ & $375(22,3)$ & $3356(27,1)$ \\
\hline \multicolumn{4}{|l|}{ Sexe $^{*}$} \\
\hline Hommes & $5468(51,0)$ & $907(54,0)$ & $6375(51,4)$ \\
\hline Femmes & $5246(49,0)$ & $773(46,0)$ & $6019(48,6)$ \\
\hline \multicolumn{4}{|l|}{ Région sanitaire $^{* *}$} \\
\hline Winnipeg & $6403(59,8)$ & $339(20,2)$ & $6742(54,4)$ \\
\hline Autre que Winnipeg & $4311(40,2)$ & $1341(79,8)$ & $5652(45,6)$ \\
\hline \multicolumn{4}{|l|}{ Quintile de revenu** } \\
\hline $\mathrm{T} 1 / \mathrm{T} 2$ zone urbaine (le plus faible) & $3079(28,7)$ & $191(11,4)$ & $3270(26,4)$ \\
\hline T3 zone urbaine & $1206(11,3)$ & $58(3,5)$ & $1264(10,2)$ \\
\hline T4/T5 zone urbaine (le plus élevé) & $2136(19,9)$ & $76(4,5)$ & $2212(17,8)$ \\
\hline T1/T2 zone rurale (le plus faible) & $1636(15,3)$ & $754(44,9)$ & $2390(19,3)$ \\
\hline T3 zone rurale & $785(7,3)$ & $192(11,4)$ & $977(7,9)$ \\
\hline T4/T5 zone rurale (le plus élevé) & $1085(10,1)$ & $347(20,7)$ & $1432(11,6)$ \\
\hline Données manquantes & $787(7,4)$ & $62(3,6)$ & $849(6,8)$ \\
\hline
\end{tabular}

Abréviations : RA, rémunéré à l'acte; NRA, non rémunéré à l'acte.

${ }^{a}$ Les pourcentages pour cette ligne sont basés sur le total de la rangée; tous les autres pourcentages du tableau sont basés sur les totaux des colonnes pour chaque variable.

$* p<0,05$.

$* * p<0,001$. 
TABLEAU 2

Caractéristiques des médecins qui ont prescrit des médicaments pour le diabète aux membres de la cohorte de l'étude, par mode de rémunération

\begin{tabular}{|c|c|c|c|}
\hline \multirow[t]{2}{*}{ Caractéristiques } & \multicolumn{3}{|c|}{ Cohorte } \\
\hline & $\begin{array}{c}\text { Médecins RA } \\
\text { n (\%) }\end{array}$ & $\begin{array}{c}\text { Médecins NRA } \\
\text { n (\%) }\end{array}$ & $\begin{array}{c}\text { Tous les médecins } \\
\text { n (\%) }\end{array}$ \\
\hline Total $^{a}$ & $959(81,8)$ & $213(18,2)$ & $1172(100,0)$ \\
\hline \multicolumn{4}{|l|}{ Groupe d'âge (ans) $^{* *}$} \\
\hline Moins de 35 & $124(12,9)$ & $38(17,8)$ & $162(13,8)$ \\
\hline $35-60$ & $689(71,9)$ & $167(78,4)$ & $856(73,0)$ \\
\hline 61 et plus & $146(15,2)$ & $8(3,8)$ & $154(13,1)$ \\
\hline \multicolumn{4}{|l|}{ Sexe } \\
\hline Hommes & $664(69,2)$ & $134(62,9)$ & $798(68,1)$ \\
\hline Femmes & $295(30,8)$ & $79(37,1)$ & $374(31,9)$ \\
\hline \multicolumn{4}{|l|}{ Spécialité** } \\
\hline Spécialiste & $211(22,0)$ & $16(7,5)$ & $227(19,4)$ \\
\hline Omnipraticien & $727(75,8)$ & $187(87,8)$ & $914(78,0)$ \\
\hline Données manquantes & $21(2,2)$ & $10(4,7)$ & $31(2,6)$ \\
\hline \multicolumn{4}{|l|}{ Région sanitaire $^{* *}$} \\
\hline Winnipeg & $645(67,3)$ & $76(35,7)$ & $721(61,5)$ \\
\hline Autre que Winnipeg & $314(32,7)$ & $137(64,3)$ & $451(38,5)$ \\
\hline
\end{tabular}

Abréviations : RA, rémunéré à l'acte; NRA, non rémunéré à l'acte.

Remarque : Une variable sans astérisque indique qu'il n'y a pas de différence statistiquement significative entre les groupes RA et NRA.

${ }^{a}$ Les pourcentages pour cette ligne sont basés sur le total de la rangée; tous les autres pourcentages du tableau sont basés sur les totaux des colonnes pour chaque variable.

$* * p<0,001$.

diabète au Manitoba au cours de la période d'étude que les médecins RA, les deux groupes de médecins différant en termes d’âge, de spécialité et de région sanitaire de pratique. Un plus grand pourcentage de

médecins NRA pratiquait dans des régions sanitaires à l'extérieur de Winnipeg plutôt que dans la région sanitaire de Winnipeg, probablement en raison de programmes provinciaux qui fournissent aux médecins

TABLEAU 3

Cohorte de l'étude avec diagnostic de diabète inclus ou non inclus par groupe d'âge et par mode de rémunération du médecin prescripteur

\begin{tabular}{lcr} 
Groupe d'âge & $\begin{array}{c}\text { Médecins RA } \\
\mathbf{n}(\%)\end{array}$ & $\begin{array}{c}\text { Cohorte } \\
\text { Médecins NRA } \\
\mathbf{n}(\%)\end{array}$ \\
\hline $\begin{array}{l}20-64 \text { ans* }^{* *} \\
\text { Présence de diagnostic }\end{array}$ & $1039(79,6)$ \\
Absence de diagnostic & $6458(83,5)$ & $266(20,4)$ \\
65 ans et plus & $1275(16,5)$ & $326(86,9)$ \\
Présence de diagnostic & $2661(89,3)$ & $49(13,1)$ \\
Absence de diagnostic & $320(10,7)$ & $1365(81,3)$ \\
Tous les âges & & $315(18,7)$ \\
Présence de diagnostic & $9119(85,1)$ & \\
Absence de diagnostic & $1595(14,9)$ & \\
\hline
\end{tabular}

Abréviations : RA, rémunéré à l'acte; NRA, non rémunéré à l'acte.

Remarques : les cas ont été classés comme inclus ou non inclus en fonction de la présence ou de l'absence d'un diagnostic de diabète dans les données de facturation des médecins et les registres de congés des hôpitaux. Un groupe d'âge sans astérisque indique qu'il n'y a pas de différence statistiquement significative entre les groupes RA et NRA.

$* * p<0,001$. d'autres modes de paiement afin de les inciter à travailler dans les régions rurales et éloignées de la province.

Lorsque nous avons utilisé les dossiers sur les médicaments d'ordonnance comme source de données de référence, nous nous sommes aperçus que les données sur le diagnostic de diabète étaient incomplètes pour les membres de la cohorte qui avaient été vus à la fois par un médecin RA et par un médecin NRA, bien que les médecins NRA soient plus nombreux à n'avoir pas inclus de diagnostic. Les causes possibles de l'absence de diagnostic sont un biais d'erreur de classification en raison d'une sensibilité imparfaite de l'information de diagnostic et un biais de données manquantes en raison du peu de médecins NRA ayant fourni des factures fictives. En supposant que la sensibilité des diagnostics de diabète soit la même pour les médecins RA et NRA, les résultats suggèrent que 3,8 \% de la facturation des médecins était manquante au Manitoba en raison d'un manque de facturation fictive. Il y avait des différences dans les pourcentages de cas non inclus par groupe d'âge pour les médecins RA et NRA, ce qui suggère une sensibilité de détermination différente entre les membres de la cohorte les plus jeunes et les membres les plus âgés.

Une étude antérieure effectuée à l'aide des données de facturation des médecins de l'Ontario ${ }^{8}$ a révélé que $23,7 \%$ des cas de diabète diagnostiqués n'apparaissaient pas dans les données de facturation des médecins NRA. Pour notre part, nous avons découvert 18,7 \% de diagnostics manquants dans les données de facturation des médecins NRA du Manitoba. L'étude de l'Ontario était axée exclusivement sur l'examen des diagnostics manquants des médecins NRA, tandis que notre étude a comparé les médecins RA et NRA. L'inclusion des médecins RA permet d'aborder la question des effets de la facturation fictive sur la proportion de diagnostics de diabète manquants dans les données de facturation des médecins. L'étude ontarienne a également utilisé des périodes de suivi variables pour saisir les diagnostics de diabète avant et après la date de l'ordonnance pour le diabète, jusqu'à un maximum de neuf ans. En 
TABLEAU 4

Caractéristiques des membres de la cohorte pour lesquels il n'y a pas de diagnostic de diabète dans les données administratives par mode de rémunération du médecin prescripteur

\begin{tabular}{|c|c|c|c|}
\hline \multirow[t]{2}{*}{ Caractéristiques } & \multicolumn{3}{|c|}{ Cohorte } \\
\hline & $\begin{array}{c}\text { Médecins RA } \\
\text { n (\%) }\end{array}$ & $\begin{array}{c}\text { Médecins NRA } \\
\text { n (\%) }\end{array}$ & $\begin{array}{c}\text { Tous les médecins } \\
\text { n (\%) }\end{array}$ \\
\hline Total $^{\mathrm{a}}$ & $1595(83,5)$ & $315(16,5)$ & $1910(100,0)$ \\
\hline \multicolumn{4}{|l|}{ Groupe d'âge (ans) $^{* *}$} \\
\hline $20-64$ & $1275(79,9)$ & $266(84,4)$ & $1541(81,0)$ \\
\hline 65 et plus & $320(20,1)$ & $49(15,6)$ & $369(19,0)$ \\
\hline \multicolumn{4}{|l|}{ Sexe $^{*}$} \\
\hline Hommes & $444(27,8)$ & $146(46,3)$ & $590(30,9)$ \\
\hline Femmes & $1151(72,2)$ & $169(53,7)$ & $1320(69,1)$ \\
\hline \multicolumn{4}{|l|}{ Région sanitaire $^{* *}$} \\
\hline Winnipeg & $847(53,1)$ & $56(17,8)$ & $903(47,3)$ \\
\hline Autre que Winnipeg & $748(46,9)$ & $259(82,2)$ & $1007(52,7)$ \\
\hline \multicolumn{4}{|l|}{ Quintile de revenu $^{* *}$} \\
\hline T1/T2 zone urbaine (le plus faible) & $411(25,8)$ & $34(10,8)$ & $445(23,3)$ \\
\hline T3 Zone urbaine & $174(10,9)$ & $10(3,2)$ & $184(9,6)$ \\
\hline T4/T5 zone urbaine (le plus élevé) & $269(16,9)$ & $7(2,2)$ & $276(14,5)$ \\
\hline $\mathrm{T} 1 / \mathrm{T} 2$ zone rurale (le plus faible) & $330(20,7)$ & $165(52,4)$ & $495(25,9)$ \\
\hline T3 zone rurale & $112(7,0)$ & $37(11,7)$ & $149(7,8)$ \\
\hline T4/T5 zone rurale (le plus élevé) & $176(11,0)$ & $53(16,8)$ & $229(12,0)$ \\
\hline Données manquantes & $123(7,7)$ & $9(2,9)$ & $132(6,9)$ \\
\hline
\end{tabular}

Abréviations : RA, rémunéré à l'acte; NRA, non rémunéré à l'acte.

Remarque : Une variable sans astérisque indique qu'il n'y a pas de différence statistiquement significative entre les groupes RA et NRA.

${ }^{a}$ Les pourcentages pour cette rangée sont basés sur le total de la rangée; tous les autres pourcentages du tableau sont basés sur les totaux des colonnes pour chaque variable.

$* p<0,05$.

$* * p<0,001$.

outre, contrairement à l'étude de l’Ontario, qui ne portait que sur la population plus âgée (65 ans et plus), nous avons inclus tous les individus âgés de 20 ans et plus et

étudié les données manquantes dans les deux groupes d'âge, les plus jeunes et les plus âgés. Nous avons constaté qu'il y avait un pourcentage plus élevé de

TABLEAU 5

Affections comorbides sélectionnées chez les individus avec diagnostic de diabète inclus et non inclus

\begin{tabular}{|c|c|c|}
\hline \multirow[t]{2}{*}{ Affection comorbide } & \multicolumn{2}{|c|}{ Cohorte } \\
\hline & Présence de diagnostic & Absence de diagnostic \\
\hline & \multicolumn{2}{|c|}{$\begin{array}{c}\text { Médecins RA } \\
\text { n (\%) }\end{array}$} \\
\hline Hypertension ${ }^{* *}$ & $662(7,3)$ & $75(4,7)$ \\
\hline \multirow[t]{2}{*}{ Maladie cardiovasculaire ${ }^{\mathrm{a},{ }^{, * *}}$} & $839(9,2)$ & $94(5,9)$ \\
\hline & \multicolumn{2}{|c|}{$\begin{array}{c}\text { Médecins NRA } \\
\text { n (\%) }\end{array}$} \\
\hline Hypertension $^{* *}$ & $100(7,3)$ & $6(1,9)$ \\
\hline Maladie cardiovasculaire ${ }^{\mathrm{a},{ }^{* *}}$ & $122(8,9)$ & $9(2,9)$ \\
\hline
\end{tabular}

Abréviations : RA, rémunéré à l'acte; NRA, non rémunéré à l'acte.

${ }^{a}$ Les maladies cardiovasculaires sont ici l'insuffisance cardiaque congestive, l'hypertension, le syndrome coronarien aigu, la maladie cérébrovasculaire (accident vasculaire cérébral) et la fibrillation auriculaire.

$* * p<0,001$. diagnostics omis dans le groupe d'âge le plus jeune, ce qui ne surprend pas puisque les personnes âgées sont plus susceptibles d'avoir une charge de morbidité plus grande et, par conséquent, d'être hospitalisées ou d'avoir des contacts réguliers avec un médecin de soins primaires.

Cette étude ayant fait la preuve d'une perte de données associée à un manque de facturation fictive par les médecins NRA, il est important d'examiner des stratégies d'adaptation des estimations de la prévalence et de l'incidence pour pallier cette sous-estimation potentielle. L'une des stratégies consiste à utiliser d'autres données basées sur la population, comme les dossiers médicaux électroniques, qui sont de plus en plus utilisés pour mener des recherches sur les maladies chroniques à l'échelle de la population et exercer une surveillance sur les maladies chroniques $^{17}$, et les sources de données sur les médicaments d'ordonnance, pour compléter les dossiers de facturation des médecins concernant la surveillance des maladies. Par exemple, en nous basant sur la définition de cas du SNSMC, nous avons estimé le taux d'incidence brut du diabète à 1,6 \% chez les 20 ans et plus au Manitoba au cours de la période d'étude. Quand on a ajouté les cas obtenus à partir des données sur les médicaments d'ordonnance pour tenir compte de la sousestimation, ce taux d'incidence est passé à $1,9 \%$.

Par ailleurs, des études de simulation et de modélisation prédictive pourraient être utilisées pour produire des estimations ajustées de la prévalence de la maladie, comme cela a été fait dans des recherches precedents $^{18,19}$. Plus précisément, nous avons constaté que les caractéristiques du patient et du médecin différaient significativement entre les groupes de médecins RA et NRA. Nous avons également constaté que la présence d'affections comorbides cardiovasculaires était associée au fait que le diagnostic de diabète du patient cadre ou non avec la définition de cas du SNSMC. Ces facteurs pourraient être inclus dans les modèles prédictifs pour estimer la prévalence de la maladie. Dans une étude récente ${ }^{20}$, nous avons inclus les caractéristiques des médecins dans les modèles prédictifs pour ajuster 
FIGURE 1

Incidence du diabète brut (\%) dans la population adulte (20 ans et plus) du Manitoba, 2007-2008 et 2008-2009

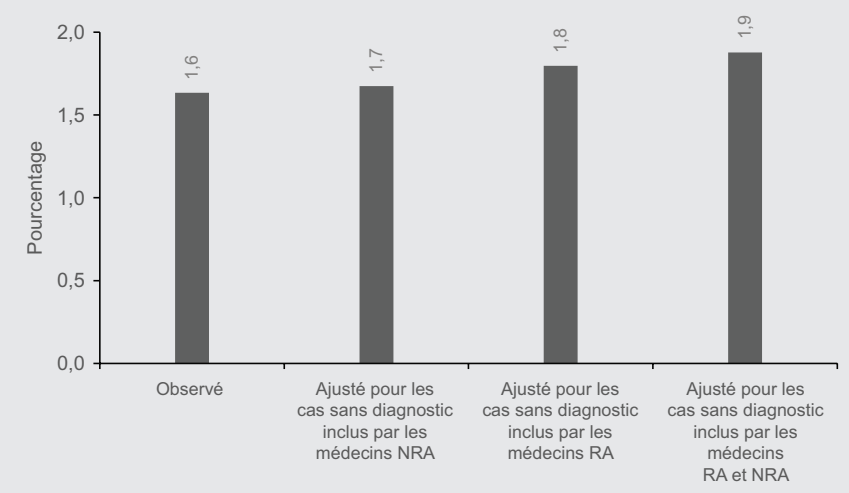

Abréviations : RA, rémunéré à l'acte; NRA, non rémunéré à l'acte.

la sous-estimation de la prévalence du diabète due à la perte de données de facturation des médecins.

\section{Points forts et limites}

Notre étude comporte certaines limites. D’abord, les médecins ont été classés comme étant soit RA soit NRA, mais certains médecins sont susceptible d'utiliser les deux types de rémunération conjointement ou de passer d'un mode de rémunération à l'autre. Étant donné que nous n'avons utilisé que les données relatives aux ordonnances pour le diabète de deux exercices, la possibilité d'un changement de mode de paiement des médecins au cours de la période d'étude est probablement minime, mais le Manitoba Provider Registry ne nous permet pas d'isoler les médecins pouvant recevoir les deux types de rémunération.

Deuxièmement, les résultats peuvent être sensibles aux définitions utilisées pour la détermination des cas omis et non omis. Nous avons fait une analyse sur une période de deux ans avant et de deux ans après l'ordonnance de référence, pour s'aligner avec la définition de cas de diabète du SNSMC utilisée dans les données administratives sur la santé. Bien qu'il soit possible que des personnes non diabétiques reçoivent une ordonnance d'un médicament pour traiter le diabète, c'est impro- bable : une recherche précédente a montré une excellente spécificité et une excellente sensibilité des dossiers de médicaments d'ordonnance pour la détermination des cas de diabète ${ }^{13}$. Enfin, les patients diabétiques dont le traitement consiste uniquement à modifier leur style de vie ne figurent pas dans les données relatives aux médicaments d'ordonnance. Le fait qu'une affection chronique puisse être traitée en modifiant seulement les habitudes de vie constitue un problème général de sousestimation de cette affection lorsqu'on utilise des données administratives pour en estimer la prévalence ou l'incidence.

\section{Conclusion}

Dans cette étude, nous avons adopté une approche basée sur la population pour évaluer l'exhaustivité des données de facturation des médecins dans le cadre de l'étude des maladies chroniques. Nous nous sommes appuyés sur des données relatives aux médicaments sur ordonnance pour en évaluer l'exhaustivité, cette source étant bien connue pour sa sensibilité dans la détermination des cas de diabète ${ }^{13}$. Notre étude a montré que la perte de données en raison de la facturation fictive omise est faible, et que cette perte de données contribue à une sous-estimation de l'incidence de la maladie. La méthode que nous avons utilisée peut être facilement appliquée à d'autres périodes et à des données provenant d'autres provinces ou territoires. Les résultats obtenus pour le diabète pourraient aussi être comparés aux résultats obtenus pour d'autres affections de santé chroniques afin d'obtenir des conclusions d'ensemble à propos de l'exhaustivité des données.

\section{Remerciements}

Les auteurs souhaitent remercier le Centre d'élaboration et d'évaluation de la politique des soins de santé du Manitoba pour l'utilisation des données contenues dans le Population Health Research Data Repository (Projet de Human Immunology Project Consortium n ${ }^{\circ}$ 2012/2013-04). Les résultats et les conclusions sont ceux des auteurs, et on ne devrait les attribuer ni officiellement ni de manière sous-entendue au Centre d'élaboration et d'évaluation de la politique des soins de santé du Manitoba, à Santé Manitoba ou aux autres fournisseurs de données.

Cette recherche est financée par les Instituts de recherche en santé du Canada ( $\mathrm{n}^{\circ}$ de référence de financement : 123357). Lisa M. Lix est titulaire d'une chaire de recherche de Recherche Manitoba. Les bailleurs de fonds n'ont joué aucun rôle dans la conduite de la recherche ou dans la préparation du manuscrit.

\section{Références}

1. Virnig BA, Mcbean M. Administrative data for public health surveillance and planning. Annu Rev Public Health. 2001;22:213-230.

2. Wasilevich Dombkowski KJ, EA, Lyon-Callo S, Nguyen Medvesky TQ, MG, Lee MA. Asthma surveillance using Medicaid administrative data: a call for a national framework. J Public Health Manag Pract. 2009;15(6): 485-93. DOI: 10.1097/PHH.0b013e3181a8c334.

3. Quan H, Khan N, Hemmelgarn BR et collab. Validation of a case definition to define hypertension using administrative data. Hypertension. 2009;54(6):1423-1428. DOI: 10.1161/HYPERTENSIONAHA.109.139279.

4. Hux JE, Ivis F, Flintoft V, Bica A. Diabetes in Ontario: determination of prevalence and incidence using a validated administrative data algorithm. Diabetes Care. 2002;25(3):512-516. 
5. Saydah SH, Geiss LS, Tierney E, Benjamin SM, Engelgau M, Brancati F. Review of the performance of methods to identify diabetes cases among vital statistics, administrative, and survey data. Ann Epidemiol. 2004; 14(7):507-516

6. Tu K, Campbell NR, Chen ZL, Cauch-Dudek KJ, McAlister FA. Accuracy of administrative databases in identifying patients with hypertension. Open Med. 2007;1(1):s18-26.

7. Gundgaard J, Ekholm O, Hansen EH, Rasmussen NK. The effect of non-response on estimates of health care utilisation: linking health surveys and registers. Eur J Public Health. 2008;18(2):189-194.

8. Alshammari AM, Hux JE. The impact of non-fee-for-service reimbursement on chronic disease surveillance using administrative data. Can J Public Heal. 2009;100(6):472-474.

9. Wranik DW, Durier-Copp M. Physician remuneration methods for family physicians in Canada: expected outcomes and lessons learned. Heal Care Anal. 2010;18(1):35-59. DOI: $10.1007 /$ s10728-008-0105-9.

10. Bernstein CN, Blanchard JF, Rawsthorne P, Wajda A, Revision N, Modification C. Epidemiology of Crohn's Disease and ulcerative colitis in a central Canadian province: a population-based study. Am J Epidemiol. 1999;149(10):916-924.

11. Robinson JR, Young TK, Roos LL, Gelskey DE, Robinson JR. Estimating the burden of disease data and self-reports administrative comparing. Med Care. 1997;35(9):932-947.

12. Lix Yogendran LM, MS, Leslie DEO et collab. Using multiple data features improved the validity of osteoporosis case ascertainment from administrative databases. J Clin Epidemiol. 2008;61(12):1250-1260.

13. Koleba T, Pohar SL, Johnson JA. Prescription drug data and the National Diabetes Surveillance System case definition. Can J Diabetes. 2007;31(1):47-53. DOI: 10.1016/ S1499-2671(07) 11010-8.

14. Clottey C, Mo F, LeBrun B, Mickelson P, Niles J, Robbins G. La mise sur pied du Système national de surveillance du diabète (SNSD) au Canada. Maladies chroniques au Canada. 2001;22(2):75-77.
15. Roos NP, Mustard CA. Variation in health and health care use by socioeconomic status in Winnipeg, Canada: does the system works well?. Yes and no. Milbank Q. 1997;75:89-111.

16. SAS Institute Inc. SAS/STAT 9.3 User's Guide. Cary (NC) : SAS Institute Inc.; 2011.

17. Desai JR, Wu P, Nichols GA, Lieu TA, O’Connor PJ. Diabetes and asthma case identification, validation, and representativeness when using electronic health data to construct registries for comparative effectiveness and epidemiologic research. Med Care. 2012;50 Suppl:S30-S35. DOI: 10.1097/MLR.0b013e318259c011.

18. Das B, Clegg LX, Feuer EJ, Pickle LW. A new method to evaluate the completeness of case ascertainment by a cancer registry. Cancer Causes Control. 2008;19(5):515-525. DOI: $10.1007 / \mathrm{s} 10552-008-9114-0$.

19. Silcocks PB, Robinson D. Simulation modelling to validate the flow method for estimating completeness of case ascertainment by cancer registries. J Public Health. 2007; 29(4):455-462.

20. Lix LM, Yao X, Kephart G et collab. A prediction model to estimate completeness of electronic physician claims databases. BMJ Open. 2015;5:e006858 DOI: 10.1136/bmjopen-2014-006858. 